\title{
Stem cell therapy in stroke
}

\author{
Sheng-Tzung Tsai', Chien-Hui Lee1, Shinn-Zong Lin', Dah-Ching Ding ${ }^{2,3}$ \\ 'Bioinnovation Center, Tzu Chi foundation, Department of Neurosurgery, Hualien Tzu Chi Hospital, Buddhist Tzu Chi Foundation, \\ Tzu Chi University, Hualien 970, Taiwan. \\ 2Department of Obstetrics and Gynecology, Hualien Tzu Chi Hospital, Buddhist Tzu Chi Foundation, Tzu Chi University, Hualien \\ 970, Taiwan. \\ ${ }^{3}$ Institute of Medical Science, College of Medicine, Tzu Chi University, Hualien 970, Taiwan.
}

Correspondence to: Dr. Dah-Ching Ding, MD, Ph.D., Department of Obstetrics and Gynecology, Hualien Tzu Chi Hospital, Buddhist Tzu Chi Medical Foundation, Tzu Chi University, No. 707, Chung-Yang Rd., Sec. 3, Hualien, 970, Taiwan, R.O.C.

E-mail: dah1003@yahoo.com.tw

How to cite this article: Tsai ST, Lee CH, Lin SZ, Ding DC. Stem cell therapy in stroke. Vessel Plus 2021;5:9.

http://dx.doi.org/10.20517/2574-1209.2020.73

Received: 17 Nov 2020 First Decision: 31 Dec 2020 Revised: 12 Jan 2021 Accepted: 19 Jan 2021 Published: 7 Feb 2021

Academic Editors: Elisa F. M. Ciceri, Aaron S. Dumont Copy Editor: Yue-Yue Zhang Production Editor: Xi-Jun Chen

\begin{abstract}
Stroke is the leading cause of neurological disability in adults worldwide and involves the significant impairment of sensory-motor function caused by cerebral ischemia and subsequent neuronal death. Owing to a lack of medical or surgical treatments to improve neurological function and neurogenesis, chronic stroke places a huge burden on patients, their families, and society. Over the past twenty years, increasing evidence from translational and clinical research has demonstrated the potential effectiveness of hematopoietic growth factors and stem cell administration or transplantation in the treatment of stroke. In particular, these studies have included granulocyte colony-stimulating factor, mesenchymal stem cells, autologous CD34 peripheral blood stem cells, umbilical cord blood stem cells, and autologous adipose-derived mesenchymal stem cells. It is therefore important to consider the safety of these putative therapies whilst achieving the maximum benefit for patients with chronic stroke in terms of the route of administration and stem cell numbers. In this review, we discuss current evidence and the progress that has been made in our hospital, which paves the way for the next neurogenesis therapy for chronic stroke.
\end{abstract}

Keywords: Stem cells, stroke, therapy, neural stem cells, mesenchymal stem cells 


\section{INTRODUCTION}

Owing to the aging global population, the socioeconomic burden of stroke is increasing, and stroke has become the second most common cause of death ${ }^{[1]}$. In Taiwan, the prevalence of stroke in those aged $>35$ years has been reported as $5.95 / 1000$ people ${ }^{[2]}$. Stroke in patients can cause long-term disability ${ }^{[3]}$, and recovery is not expected within five months ${ }^{[4]}$. After this period, surviving patients are regarded as having had a chronic stroke and require long-term rehabilitation ${ }^{[5]}$. Although mobility can be partially recovered, neurological deficits resulting from neuronal loss are difficult to repair ${ }^{[4]}$; therefore, novel treatment methods are urgently required.

Patients with acute stroke are generally treated using thrombolysis intravenously with tissue plasminogen activator (tPA) within $3 \mathrm{~h}^{[6]}$; however, thrombolysis therapy can cause complications such as intracranial hemorrhage, angioedema, and major systemic hemorrhage in $6 \%, 5 \%$, and $2 \%$ of patients, respectively ${ }^{[7]}$. Concomitant rehabilitation is important for stroke recovery as it can reduce disability, promote recovery, and improve quality of $\operatorname{life}^{[5]}$. Despite investigations into transcranial magnetic stimulation, there is no definitive evidence of the efficacy of this approach, while the administration of ion-channel mediators (e.g., dalfampridine) and catecholamine agonists (e.g., amphetamine) has proven to be ineffective ${ }^{[8]}$.

Owing to the current shortage of effective therapies, it is important to develop novel therapeutic modalities. For instance, granulocyte colony-stimulating factor (GCSF) has been reported to modestly improve outcomes by enhancing the generation of endogenous stem cells ${ }^{[9]}$. A growing body of evidence has shown that stem cell therapy could help to reconstruct neuronal circuits after chronic stroke ${ }^{[10]}$; therefore, exogenous stem cell transplantation could underlie the next generation of therapies for patients suffering from stroke. In this review, we discuss current evidence and the progress of stem cell therapy in stroke patients that has been made in our hospital (Hualien Tzu Chi Hospital, Hualien, Taiwan).

\section{STEM CELL THERAPY IN STROKE}

Stem cell therapies for stroke aim to repair, replace, and enhance the biological function of damaged or dead cells to restore neural integrity ${ }^{[10]}$. For instance, differentiated neuronal progenitor cells may repair the functional neurons circuitry, and the paracrine factors that they secrete can promote the survival, differentiation, and migration of endogenous penumbral progenitor cells ${ }^{[11]}$. Until now, the majority of clinical trials for stroke have used cultured autologous mesenchymal stem cells (MSCs) derived from bone marrow, adipose tissue, or umbilical cord ${ }^{[10]}$ administered via intravenous transplantation, which is a simple delivery technique that has been approved by government regulations ${ }^{[10]}$.

\section{PRECLINICAL MODELS}

The variants of MSCs include umbilical cord blood (UCB) mononuclear cells (MNCs), human umbilical cord mesenchymal stem cells (HUCMSCs), bone marrow stem cells (BMSCs), and adipose tissue-derived stem cells (ADSCs), whereas endogenous stem cells include peripheral blood CD34 cells, GCSF-induced CD34 cells, and neural stem cells (NSCs) ${ }^{[12]}$. Each different type of stem cell has a different effect on stroke recovery; however, MSCs are currently the most widely used cell type for stroke therapy ${ }^{[10,11]}$. In this section, we introduce different types of stem cells used in preclinical research.

\section{Umbilical cord blood mononuclear cells}

Human UCB contains numerous hematopoietic and endothelial primitive cells that display strong replication capacities in vitro and in vivo. In addition, the high levels of EGF, VEGF, G-CSF, and IL-10 in UCB suggest that treatment with UCB could both restore immune homeostasis and enhance the repair of damaged neurons in patients with cerebral stroke ${ }^{[13]}$. Human UCB MNCs have been shown to exert robust therapeutic effects in experimental models of acute and subacute stroke. For example, intravenous 
UCB MNC injection was found to restore impaired exercise capabilities and exert neuroprotective effects in rodent models of cerebral stroke ${ }^{[13-15]}$. Moreover, UCB MNCs have immunomodulatory and antiinflammatory effects in addition to their regenerative effects and can, therefore, protect penumbral tissue from further injury caused by inflammation after stroke ${ }^{[16]}$.

MNCs can also exert immunomodulatory effects by changing the phenotype of splenocytes ${ }^{[17]}$; therefore, intravenous MNC therapy may achieve neural protection by altering systemic immunomodulation in the acute or subacute stages of stroke. UCB serum was also found to contain increased heat shock protein 27 levels and exert anti-senescent effects in implanted stem cells to increase stem cell engraftment and differentiation $^{[18]}$. Importantly, MNCs and other adult tissue-derived stem cells can be easily isolated and expanded in the laboratory, while the safety profile of MNCs has been consistently demonstrated in clinical trials ${ }^{[13,14]}$. A recent Phase I study was the first to demonstrate that a single intravenous administration of allogenic non-HLA matched human UCB cells is beneficial and safe for patients with acute ischemic stroke $^{[16]}$. Therefore, UCB could be a source of allogeneic stem cells to treat acute cerebral infarction.

\section{Human umbilical cord matrix MSCs}

Human umbilical cord (HUC) is an emerging source of MSCs (HUCMSCs), which harbor rapid renewal properties and can be acquired through painless collection procedures. HUCMSCs can be derived from the cord lining, Wharton's jelly, and perivascular tissue, and they can be easily differentiated into three germ layers that promote tissue repair. Previously, we found that HUCMSCs can improve both functional angiogenesis and neuroplasticity in animal models of stroke by upregulating $\beta 1$-integrin ${ }^{[18,19]}$. In addition, HUCMSCs can be differentiated into microglial cells; they can also be induced to produce neuronal proteins and increase astrocyte protein glial fibrillary acidic protein level ${ }^{[20]}$. Furthermore, a three-dimensional alginate scaffold has been used to effectively induce HUCMSCs to become neurons ${ }^{[21]}$. Therefore, HUCMSCs could be a source of allogeneic stem cells to treat acute cerebral infarction.

\section{Hematopoietic factor and endogenous stem cell mobilization}

GCSF is a growth factor that belongs to a cytokine family. GCSF can mobilize hematopoietic stem cells from bone marrow into peripheral blood. GCSF was routinely used to treat neutropenia and to reconstitute the bone marrow; however, hematopoietic stem cells in transplantation have recently been replaced by bone marrow to regenerate non-hematopoietic tissues in conditions such as myocardial infarction (MI). GCSF administration after MI has been shown to improve cardiac function and survival rates ${ }^{[22]}$, while its antiinflammatory and anti-apoptotic effects can help prevent neuronal and glial pro-inflammatory cascades, which have both been implicated in the pathophysiology of chronic ischemic injury of the brain ${ }^{[23]}$. Activated neutrophils can produce microvascular plugging and cytotoxic substances, which will cause focal cerebral ischemic infarction. Thus, reducing inflammation in the stroke area may preserve against ischemic injury of the brain. GCSF can decrease excitotoxicity in cell culture and reduce programmed cell death in rats with cerebral ischemia ${ }^{[24]}$. Furthermore, a seminal paper confirms that GCSF reduced the infarction area and improved the recovery of rats with stroke by enhancing angiogenesis and neurogenesis ${ }^{[2,25]}$. Therefore, GCSF could be used as a therapy for acute and chronic stroke.

\section{MSCs and peripheral hematopoietic stem cells (CD34 $)$}

Owing to the efficacy of GCSF, the potential of peripheral hematopoietic stem cells (PBSCs) for treating acute and chronic cerebral ischemia was explored since the clinical use of embryonic stem cells is hindered by ethical issues. In stem cell therapy for stroke, PBSCs are regarded as a source of hematopoietic stem cells and are increasingly being employed for transplantation. GCSF can mobilize PBSCs from bone marrow and increase their numbers ${ }^{[26]}$. Indeed, intracerebral PBSC transplantation in rats with chronic cerebral ischemia has been shown to greatly improve neurological function ${ }^{[26,27]}$. The repair mechanisms involve macrophage/ microglial cells derived from stem cells and the expression of $\beta 1$ integrin to facilitate angiogenesis in the injured brain area. 
Although the intracerebral implantation of PBSCs has shown efficacy in experimental models of stroke, this strategy is highly invasive ${ }^{[28,29]}$, and other studies have also improved neurological function in animal models using MSCs delivered via intravenous or intra-arterial routes ${ }^{[30,31]}$. In particular, bone marrow mesenchymal stem cells reduced apoptosis and neuro-inflammation around the infarct region and enhanced angiogenesis by accumulating extensively around the infarct area and differentiating into neuronal or glial cells, as confirmed using immunohistochemical studies ${ }^{[32]}$. Therefore, MSCs could be a source of autologous stem cells to treat acute and chronic cerebral infarction.

As one of the extensively used stem cells with proven safety, there is increasing need to explore the therapeutic potential of genetically-engineered MSCs with the aim to expand its application and increase its efficiency of integration into stroke lesions. The advantages of MSCs include the ability to locate themselves to the lesioned area, rapid proliferation, anti-inflammatory effects, immunomodulatory capability, strong release of paracrine or growth factors, and the potential to differentiate and integrate into various cell types and tissues. Through genetic engineering methods, MSCs could be modified by insertion of genes and their expression through viral gene transduction or a non-viral way ${ }^{[3,34]}$. This could further maximize the treatment efficacy with adequate numbers of MSCs. For example, human MSCs transfected with the $B D N F$ gene through adenovirus enhanced functional recovery of stroke in rodents ${ }^{[35]}$. Geneticallyengineered MSCs were found to have about 23-fold increased expression of BDNF, which was regarded as one of the key growth factors not only to enable neuroprotection after stroke but also to promote neuronal differentiation and repair of damaged brain.

\section{Neural stem cells}

Increasing evidence has also demonstrated the effect of NSCs in animal models of stroke. For instance, the implantation of intraparenchymal NSCs around the infarct region was found to improve sensorimotor dysfunction and motor disability ${ }^{[32,36]}$, while further analysis of the infarct area suggested that lesion topology plays an important role in functional recovery after NSC transplantation, particularly improving outcomes for strokes confined to the striatal area. This also highlights the importance of using a novel celldelivery method with instruments to maximize the treatment effectiveness of NSC implantation. Zhang et al. ${ }^{[37,38]}$ recently showed that the intracerebral microinjection instrument could deliver unprecedented numbers of NSCs to enable better three-dimensional distribution around stroke lesions. NSC transplantation in cerebral ischemia may contribute hypoxia-inducible factor-1alpha ${ }^{[39]}$, while the cell-dose dependent effect of NSCs on functional recovery suggests that a high number of stem cells is crucial to ensure adequate neurogenesis and angiogenesis ${ }^{[40]}$. Above all, NSCs could be used for treating cerebral infarction.

\section{STEM CELL THERAPY IN CLINICAL TRIALS AT HUALIEN TZU CHI HOSPITAL}

We previously performed several clinical trials of stem cell therapy for stroke patients. In this section, we introduce GCSF, CD $34^{+}$, and ADSC used as the treatment of stroke in the clinical trials in our hospital.

\section{GCSF}

Based on its capacity to mobilize stem cells and its anti-inflammatory and neuroprotective properties, GCSF can be potentially used in a clinical setting to treat several conditions, including the treatment of MI and stroke. Experimental models of chronic stroke have revealed the synergistic benefit of using GCSF to stimulate the mobilization of hematopoietic stem cells via mechanisms involving increased cerebral blood flow, survival signal transduction, and the mobilization and differentiation of endogenous stem cells; however, the efficacy of GCSF in stroke was unclear until relatively recently.

A randomized controlled trial first demonstrated the efficacy of using GCSF to treat patients with acute ischemic stroke ${ }^{[9]}$, reporting significant improvements in neurological function according to the National Institutes of Health Stroke Scale, European Stroke Scale, and Barthel Index. Several studies 
have subsequently explored the benefits of GCSF in acute ischemic stroke; however, the extent of the improvements caused by GCSF may vary between patients due to hemorrhagic infarct transformation, alterations in the blood-brain barrier, overly astrogliosis, and polarized immune cell alteration after GCSF administration ${ }^{[9,41,42]}$. In addition, dose-dependent immunomodulation has been shown to alter the central nervous system-specific effects of GCSF after stroke; therefore, optimizing the dose or treatment time may help overcome this drawback and provide consistent efficacy during the use of GCSF ${ }^{[43]}$. Above all, GCSF is successfully used in the treatment of stroke patients.

\section{Bone marrow MSCs (CD34 $4^{+}$}

Given the preclinical evidence demonstrating the additional benefits of intracerebral PBSC implantation in animal models of chronic stroke, we initiated studies to explore the benefits of PBSCs. Among 30 chronic stroke patients, GCSF-treated group and stereotactic PBSC implantation displayed the most improvement in all functional characteristics ${ }^{[28]}$. Furthermore, MRI diffusion tensor imaging revealed that the implanted PBSCs ameliorated fiber tract disconnection caused by cerebral ischemia, and motor-evoked potential via transcranial magnetic stimulation was shown to establish neurophysiological re-connection. Therefore, $\mathrm{CD} 34^{+}$mobilized by GCSF is successfully used in the treatment of stroke patients.

\section{MSC and adipose-derived stem cells}

MSCs are the most common stem cells used to treat stroke, with bone marrow- and adipose tissue-derived stem cells being the most explored cell sources ${ }^{[44]}$, since autologous stem cells can be used to treat acute or chronic stroke without carcinogenicity, immune rejection, or ethical concerns ${ }^{[45]}$. In particular, ADSCs can be obtained from subcutaneous adipose tissue fragments or liposuction aspirates easily. ADSCs can be expanded in vitro, and there are no ethical concerns regarding various clinical applications. The autologous ADSCs administration into the injured penumbra for chronic stroke patients was shown to result in functional regeneration ${ }^{[46,47]}$; therefore, we undertook the first Phase I clinical study using the stereotactic implantation of autologous ADSCs to treat patients with chronic stroke at Hualien Tzu Chi Hospital in Taiwan. Our preliminary results indicate good safety and significantly improved sensorimotor function in the study participants. Above all, ADSC could be safely and effectively used in the treatment for stroke patients.

\section{ADMINISTRATIVE PATHWAY AND TRANSPLANTED CELL NUMBERS}

Previous clinical studies have utilized several different cell-transplantation techniques. For instance, in the MultiStem study, patients were administered 1200 million cells via intravenous (IV) injection ${ }^{[48]}$. Although IV route is considered noninvasive and facilitates drug administration, some preclinical studies have suggested that most of the stem cells in circulation will be trapped in the lungs and liver, thus stem cells were not able to cross the blood-brain barrier ${ }^{[49]}$. Moreover, chemotactic signaling from the injured brain areas is not active during chronic strokes ${ }^{[50]}$. The primary mechanism of intravenous circulated stem cells may be contributed by reducing immune response in the stroke subacute phase. A Phase II trial of subacute stroke has reported that intravenous administration of bone marrow-derived mononuclear cells is safe but does not affect measures of neurological function ${ }^{[51]}$.

Stem cells can be administered by intra-arterial route to transfer into the brain directly; however, there is concern that this method could result in possible cell clumping, leading to microthrombi and potential damage ${ }^{[52]}$. Sung et al ${ }^{[53]}$ conducted a Phase I clinical trial in which circulation-derived autologous CD $34^{+}$ cells $\left(3.0 \times 10^{7}\right.$ cells/patient) were isolated and administered via intra-carotid artery transfusion to elderly patients with chronic ischemic stroke. To avoid microembolization, the CD $34^{+}$cells were first filtered with an embolic protection filter, slowly transfused into the ipsilateral internal carotid artery, and finally into the infarct area ${ }^{[53]}$. Although the neurological recovery achieved by intracerebroventricular (ICV) ADSC administration was found to be superior to that achieved via the IV rout ${ }^{[54]}$, the invasive procedure to 


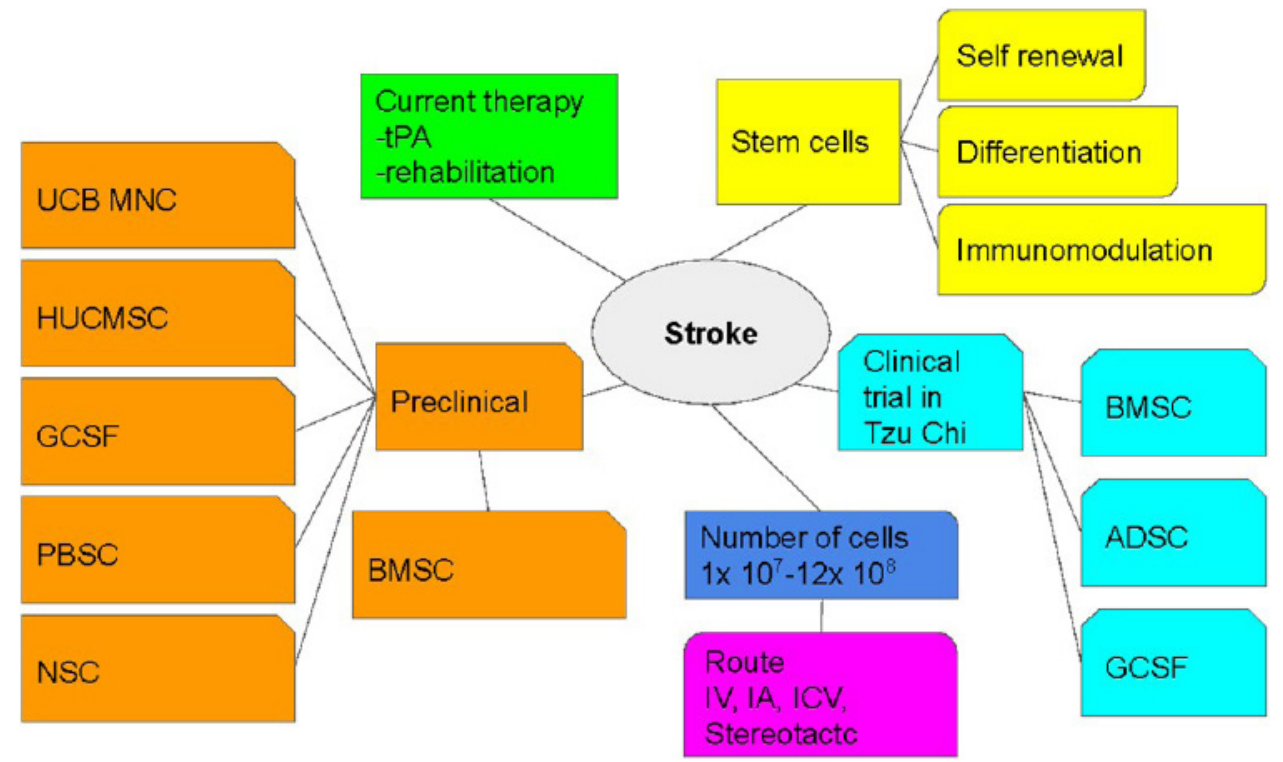

Figure 1. The landscape of this review. tPA: Tissue plasminogen activator; UCB MNC: umbilical cord blood mononuclear cell; HUCMSC: human umbilical cord mesenchymal stem cells; GCSF: granulocyte colony-stimulating factor; PBSC: peripheral blood stem cell; NSC: neural stem cell; BMSC: bone marrow stem cell; ADSC: adipose stem cell; IV: intravenous; IA: intra-arterial: ICV: intracerebroventricular.

deliver stem cells may cause surgical complications or intracerebral trauma. Thus, ICV-transplanted stem cells could be used to attract IV administered stem cells by its concentration slope of homing factors. Therefore, the route of administration and stem cell numbers are important factors for stem cell therapy in stroke patients.

\section{PERSPECTIVES}

Although stroke is currently treated using tPA and rehabilitation, new therapies are constantly being developed, among which stem cell therapies are the most promising. The variants of MSCs include UCBMSCs, HUCMSCs, BMSCs, and ADSCs, whereas endogenous stem cells include peripheral blood CD34 cells, GCSF-induced CD34 cells, and NSCs ${ }^{[12]}$. Each different type of stem cell has a different effect on stroke recovery; however, MSCs are currently the most widely used cell type for stroke therapy ${ }^{[10,11]}$.

Stem cell therapies have several advantages, including self-renewal, differentiation, and immunomodulatory capabilities. MSCs display self-renewal capabilities. A previous study tested this phenomenon ${ }^{[5]}$ and reported that single cell-derived clones display different self-renewal potentials and, thus, may harbor different differentiation capacities. In particular, MSCs can differentiate into adipocytes, osteocytes, chondrocytes, and neuron-like cells ${ }^{[56]}$, depending on the factors that they are exposed to, suggesting that MSCs could be used to treat stroke.

IV and stereotactic stem cell transplantation are commonly used routes of administration when treating stroke; however, IV is used most often. In our hospital, we used stereotactic transplantation based on the site of injection; however, this method may require specific training. There is currently no consensus regarding the number of transplanted stem cells, since too many cells can cause volume-compression effects and too few cells can be ineffective. While previous trials have used $1 \times 10^{7}$ to $12 \times 10^{8}$ cells, we transplanted $1 \times 10^{8}$ cells in our clinical trial and demonstrated good preliminary safety; however, the dosage may vary due to stroke volume and site. The landscape of this review is summarized in Figure 1. 


\section{CONCLUSIONS}

Stroke is the leading global cause of adult neurological disability, which causes sensory-motor function impairment and neuronal death; however, there are currently no medical or surgical treatments to improve neurological function and neurogenesis in chronic stroke. Growing translational and clinical evidence has demonstrated the potential effectiveness of hematopoietic growth factors and stem cell transplantation as therapies for stroke, including GCSF, mesenchymal stem cells, autologous CD $34^{+}$peripheral blood stem cells, umbilical cord blood and stromal stem cells, and autologous adipose-derived mesenchymal stem cells. This review not only highlights the safety aspects but also indicates how to achieve the maximum benefit for patients with chronic stroke by considering the route of administration and stem cell numbers. Therefore, these studies pave the way for the next generation of neurogenesis therapies for chronic stroke.

\section{DECLARATIONS}

\section{Authors' contributions}

Literature review: Tsai ST, Ding DC

Writing the manuscript: Tsai ST, Lee CH, Lin SZ, Ding DC

Organizing the manuscript: Ding DC

Discussion of the manuscript: Tsai ST, Lee CH, Ding DC, Lin SZ

\section{Availability of data and materials}

Not applicable.

\section{Financial support and sponsorship}

The work was supported by the Buddhist Tzu Chi Medical Foundation (TCMF-EP-108-02 to DCD, TCMFCWP-109-01, TCMF-MP 108-01-03, Buddhist Tzu Chi Medical Foundation).

\section{Conflicts of interest}

All authors declared that there are no conflicts of interest.

\section{Ethical approval and consent to participate}

Not applicable.

\section{Consent for publication}

Not applicable.

\section{Copyright}

(c) The Author(s) 2021.

\section{REFERENCES}

1. Feigin VL, Lawes CM, Bennett DA, Barker-collo SL, Parag V. Worldwide stroke incidence and early case fatality reported in 56 population-based studies: a systematic review. Lancet Neurol 2009;8:355-69.

2. Huang ZS, Chiang TL, Lee TK. Stroke prevalence in Taiwan. Findings from the 1994 National Health Interview Survey. Stroke 1997;28:1579-84.

3. Feigin VL, Lawes CM, Bennett DA, Anderson CS. Stroke epidemiology: a review of population-based studies of incidence, prevalence, and case-fatality in the late 20th century. Lancet Neurol 2003;2:43-53.

4. Jørgensen HS, Nakayama H, Raaschou HO, Vive-larsen J, Støier M, Olsen TS. Outcome and time course of recovery in stroke. Part II: Time course of recovery. The copenhagen stroke study. Arch Phys Med Rehabil 1995;76:406-12.

5. Winstein CJ, Stein J, Arena R, et al; American Heart Association Stroke Council, Council on Cardiovascular and Stroke Nursing, Council on Clinical Cardiology, and Council on Quality of Care and Outcomes Research. Guidelines for Adult Stroke Rehabilitation and Recovery: A Guideline for Healthcare Professionals From the American Heart Association/American Stroke Association. Stroke 2016;47:e98-e169. 
6. Powers WJ, Rabinstein AA, Ackerson T, et al; American Heart Association Stroke Council. 2018 Guidelines for the Early Management of Patients With Acute Ischemic Stroke: A Guideline for Healthcare Professionals From the American Heart Association/American Stroke Association. Stroke 2018;49:e46-e110.

7. Chugh C. Acute ischemic stroke: management approach. Indian J Crit Care Med 2019;23:S140-6.

8. Yeo SH, Lim ZI, Mao J, Yau WP. Effects of central nervous system drugs on recovery after stroke: a systematic review and meta-analysis of randomized controlled trials. Clin Drug Investig 2017;37:901-28.

9. Shyu WC, Lin SZ, Lee CC, Liu DD, Li H. Granulocyte colony-stimulating factor for acute ischemic stroke: a randomized controlled trial. CMAJ 2006;174:927-33.

10. Chang YH, Wu KC, Harn HJ, Lin SZ, Ding DC. Exosomes and Stem Cells in Degenerative Disease Diagnosis and Therapy. Cell Transplant 2018;27:349-63.

11. Ding DC, Shyu WC, Lin SZ, Li H. Current concepts in adult stem cell therapy for stroke. Curr Med Chem 2006;13:3565-74.

12. Ding DC, Lin CH, Shyu WC, Lin SZ. Neural stem cells and stroke. Cell Transplant 2013;22:619-30.

13. Ehrhart J, Sanberg PR, Garbuzova-Davis S. Plasma derived from human umbilical cord blood: Potential cell-additive or cell-substitute therapeutic for neurodegenerative diseases. J Cell Mol Med 2018;22:6157-66.

14. Zheng H, Zhang B, Chhatbar PY, et al. Mesenchymal Stem Cell Therapy in Stroke: A Systematic Review of Literature in Pre-Clinical and Clinical Research. Cell Transplant 2018;27:1723-30.

15. Vendrame M, Cassady J, Newcomb J, et al. Infusion of human umbilical cord blood cells in a rat model of stroke dose-dependently rescues behavioral deficits and reduces infarct volume. Stroke 2004;35:2390-5.

16. Sanberg PR, Ehrhart J. A Hallmark Clinical Study of Cord Blood Therapy in Adults with Ischemic Stroke. Cell Transplant 2019;28:132932.

17. Vendrame M, Gemma C, Pennypacker KR, et al. Cord blood rescues stroke-induced changes in splenocyte phenotype and function. Exp Neurol 2006;199:191-200.

18. Liu SP, Ding DC, Wang HJ, et al. Nonsenescent Hsp27-upregulated MSCs implantation promotes neuroplasticity in stroke model. Cell Transplant 2010;19:1261-79.

19. Ding DC, Shyu WC, Chiang MF, et al. Enhancement of neuroplasticity through upregulation of beta1-integrin in human umbilical cordderived stromal cell implanted stroke model. Neurobiol Dis 2007;27:339-53.

20. Fu YS, Shih YT, Cheng YC, Min MY. Transformation of human umbilical mesenchymal cells into neurons in vitro. J Biomed Sci 2004;11:652-60.

21. Hosseini SM, Vasaghi A, Nakhlparvar N, Roshanravan R, Talaei-Khozani T, Razi Z. Differentiation of Wharton’s jelly mesenchymal stem cells into neurons in alginate scaffold. Neural Regen Res 2015;10:1312-6.

22. Fujita J, Mori M, Kawada H, et al. Administration of granulocyte colony-stimulating factor after myocardial infarction enhances the recruitment of hematopoietic stem cell-derived myofibroblasts and contributes to cardiac repair. Stem Cells 2007;25:2750-9.

23. Solaroglu I, Cahill J, Tsubokawa T, Beskonakli E, Zhang JH. Granulocyte colony-stimulating factor protects the brain against experimental stroke via inhibition of apoptosis and inflammation. Neurol Res 2009;31:167-72.

24. Kong T, Choi JK, Park H, et al. Reduction in programmed cell death and improvement in functional outcome of transient focal cerebral ischemia after administration of granulocyte-macrophage colony-stimulating factor in rats. Laboratory investigation. $J$ Neurosurg 2009;111:155-63.

25. Shyu WC, Lin SZ, Yang HI, et al. Functional recovery of stroke rats induced by granulocyte colony-stimulating factor-stimulated stem cells. Circulation 2004;110:1847-54.

26. Demetri GD, Griffin JD. Granulocyte colony-stimulating factor and its receptor. Blood 1991;78:2791-808.

27. Shyu WC, Lin SZ, Chiang MF, Su CY, Li H. Intracerebral peripheral blood stem cell (CD34 $\left.{ }^{+}\right)$implantation induces neuroplasticity by enhancing betal integrin-mediated angiogenesis in chronic stroke rats. $J$ Neurosci 2006;26:3444-53.

28. Chen DC, Lin SZ, Fan JR, et al. Intracerebral implantation of autologous peripheral blood stem cells in stroke patients: a randomized phase II study. Cell Transplant 2014;23:1599-612.

29. Song M, Mohamad O, Gu X, Wei L, Yu SP. Restoration of intracortical and thalamocortical circuits after transplantation of bone marrow mesenchymal stem cells into the ischemic brain of mice. Cell Transplant 2013;22:2001-15.

30. Brenneman M, Sharma S, Harting M, et al. Autologous bone marrow mononuclear cells enhance recovery after acute ischemic stroke in young and middle-aged rats. J Cereb Blood Flow Metab 2010;30:140-9.

31 Kamiya N, Ueda M, Igarashi H, et al. Intra-arterial transplantation of bone marrow mononuclear cells immediately after reperfusion decreases brain injury after focal ischemia in rats. Life Sci 2008;83:433-7.

32. Iihoshi S, Honmou O, Houkin K, Hashi K, Kocsis JD. A therapeutic window for intravenous administration of autologous bone marrow after cerebral ischemia in adult rats. Brain Res 2004;1007:1-9.

33. Nowakowski A, Walczak P, Janowski M, Lukomska B. Genetic Engineering of Mesenchymal Stem Cells for Regenerative Medicine. Stem Cells Dev 2015;24:2219-42.

34. Hwang BW, Kim SJ, Park KM, et al. Genetically engineered mesenchymal stem cell therapy using self-assembling supramolecular hydrogels. J Control Release 2015;220:119-29.

35. Kurozumi K, Nakamura K, Tamiya T, et al. BDNF gene-modified mesenchymal stem cells promote functional recovery and reduce infarct size in the rat middle cerebral artery occlusion model. Mol Ther 2004;9:189-97.

36 Smith EJ, Paul Stroemer RP, Gorenkova N, et al. Implantation site and lesion topology determine efficacy of a human neural stem cell line in a rat model of chronic stroke. Stem Cells 2012;30:785-96. 
37. Zhang G, Li Y, Reuss JL, et al. Stable Intracerebral Transplantation of Neural Stem Cells for the Treatment of Paralysis Due to Ischemic Stroke. Stem Cells Transl Med 2019;8:999-1007.

38. Zhang G, Cunningham M, Zhang H, et al. First Human Trial of Stem Cell Transplantation in Complex Arrays for Stroke Patients Using the Intracerebral Microinjection Instrument. Oper Neurosurg (Hagerstown) 2020;18:503-10.

39. Wu W, Chen X, Hu C, Li J, Yu Z, Cai W. Transplantation of neural stem cells expressing hypoxia-inducible factor-1alpha (HIF-1alpha) improves behavioral recovery in a rat stroke model. J Clin Neurosci 2010;17:92-5.

40 Stroemer P, Patel S, Hope A, Oliveira C, Pollock K, Sinden J. The neural stem cell line CTX0E03 promotes behavioral recovery and endogenous neurogenesis after experimental stroke in a dose-dependent fashion. Neurorehabil Neural Repair 2009;23:895-909.

41. Strecker JK, Olk J, Hoppen M, et al. Combining Growth Factor and Bone Marrow Cell Therapy Induces Bleeding and Alters Immune Response After Stroke in Mice. Stroke 2016;47:852-62.

42. England TJ, Sprigg N, Alasheev AM, et al. Granulocyte-Colony Stimulating Factor (G-CSF) for stroke: an individual patient data metaanalysis. Sci Rep 2016;6:36567.

43. Wagner DC, Pösel C, Schulz I, et al. Allometric dose retranslation unveiled substantial immunological side effects of granulocyte colonystimulating factor after stroke. Stroke 2014;45:623-6.

44. Singh M, Pandey PK, Bhasin A, Padma MV, Mohanty S. Application of Stem Cells in Stroke: A Multifactorial Approach. Front Neurosci 2020;14:473.

45. Ding DC, Shyu WC, Lin SZ. Mesenchymal stem cells. Cell Transplant 2011;20:5-14.

46. Scruggs BA, Semon JA, Zhang X, et al. Age of the donor reduces the ability of human adipose-derived stem cells to alleviate symptoms in the experimental autoimmune encephalomyelitis mouse model. Stem Cells Transl Med 2013;2:797-807.

47. Gutiérrez-Fernández M, Rodríguez-Frutos B, Otero-Ortega L, Ramos-Cejudo J, Fuentes B, Díez-Tejedor E. Adipose tissue-derived stem cells in stroke treatment: from bench to bedside. Discov Med 2013;16:37-43.

48. Hess DC, Sila CA, Furlan AJ, Wechsler LR, Switzer JA, Mays RW. A double-blind placebo-controlled clinical evaluation of MultiStem for the treatment of ischemic stroke. Int J Stroke 2014;9:381-6.

49. Vahidy FS, Rahbar MH, Zhu H, Rowan PJ, Bambhroliya AB, Savitz SI. Systematic Review and Meta-Analysis of Bone Marrow-Derived Mononuclear Cells in Animal Models of Ischemic Stroke. Stroke 2016;47:1632-9.

50. Chan TM, Harn HJ, Lin HP, et al. The use of ADSCs as a treatment for chronic stroke. Cell Transplant 2014;23:541-7.

51. Prasad K, Sharma A, Garg A, et al; InveST Study Group. Intravenous autologous bone marrow mononuclear stem cell therapy for ischemic stroke: a multicentric, randomized trial. Stroke 2014;45:3618-24.

52. Watanabe M, Yavagal DR. Intra-arterial delivery of mesenchymal stem cells. Brain Circ 2016;2:114-7.

53. Sung PH, Lin HS, Lin WC, et al. Intra-carotid arterial transfusion of autologous circulatory derived CD $34^{+}$cells for old ischemic stroke patients - a phase I clinical trial to evaluate safety and tolerability. Am J Transl Res 2018;10:2975-89.

54. Wang Y, Huang J, Li Y, Yang GY. Roles of chemokine CXCL12 and its receptors in ischemic stroke. Curr Drug Targets 2012;13:166-72.

55. Sarugaser R, Hanoun L, Keating A, Stanford WL, Davies JE. Human mesenchymal stem cells self-renew and differentiate according to a deterministic hierarchy. PLoS One 2009;4:e6498.

56. Hernández R, Jiménez-Luna C, Perales-Adán J, Perazzoli G, Melguizo C, Prados J. Differentiation of Human Mesenchymal Stem Cells towards Neuronal Lineage: Clinical Trials in Nervous System Disorders. Biomol Ther (Seoul) 2020;28:34-44. 